\title{
Design of Optimal RF Pulses for NMR as a Discrete-Valued Control Problem *
}

\author{
Christian Clason* Carla Tameling ** Benedikt Wirth*** \\ * Faculty of Mathematics, Universität Duisburg-Essen, 45117 Essen, \\ Germany (e-mail: christian.clason@uni-due.de) \\ ** Institute for Mathematical Stochastics, Universität Göttingen, \\ Goldschmidtstr. 7, 3707 röttingen, Germany (e-mail: \\ carla.tameling@mathematik.uni-goettingen.de) \\ *** Applied Mathematics, Universität Münster, Einsteinstr. 62, 48149 \\ Münster, Germany (e-mail: benedikt.wirth@uni-muenster.de)
}

Keywords: multibang control, convex relaxation, semismooth Newton, Bloch equation, NMR

\section{INTRODUCTION}

Designing optimal radiofrequency (RF) pulses for nuclear magnetic resonance (NMR) imaging consists of driving a collection of spin systems using external electromagnetic fields of minimal energy to a desired spin state. However, in some cases the hardware only allows a discrete set of pulse phases and amplitudes. In contrast to methods based on quantization (Dridi et al., 2015), we aim to compute such pulses by solving a suitable optimal control problem.

A standard model for NMR is given by the Bloch equation in a rotating reference frame without relaxation, i.e.,

$$
\frac{\mathrm{d}}{\mathrm{d} t} \mathbf{M}^{(\omega)}(t)=\mathbf{M}^{(\omega)}(t) \times \mathbf{B}^{(\omega)}(t), \quad \mathbf{M}^{(\omega)}(0)=\mathbf{M}_{0},
$$

which describes the temporally evolving magnetization $\mathbf{M}^{(\omega)} \in \mathbb{R}^{3}$ of an ensemble of spins rotating at the same resonance offset frequency $\omega$ (called isochromat), starting from a given equilibrium magnetization $\mathbf{M}_{0} \in \mathbb{R}^{3}$ (e.g., aligned to a strong external field). The time-varying effective magnetic field $\mathbf{B}^{(\omega)}(t)$ is of the form

$$
\mathbf{B}^{(\omega)}(t)=\left(\omega_{x}(t), \omega_{y}(t), \omega\right)^{T}
$$

where $u(t):=\left(\omega_{x}(t), \omega_{y}(t)\right) \in \mathbb{R}^{2}$ can be controlled. The aim is to achieve a magnetization $\mathbf{M}^{(\omega)}(T)=\mathbf{M}_{d}^{(\omega)}$ within the time interval $\Omega=[0, T]$ for a list of offset frequencies $\omega_{1}, \ldots, \omega_{J}$ using control values $u(t)$ from a discrete set

$$
\mathcal{M}=\left\{\left(\begin{array}{l}
0 \\
0
\end{array}\right),\left(\begin{array}{c}
\omega_{0} \cos \theta_{1} \\
\omega_{0} \sin \theta_{1}
\end{array}\right), \ldots,\left(\begin{array}{c}
\omega_{0} \cos \theta_{M} \\
\omega_{0} \sin \theta_{M}
\end{array}\right)\right\}
$$

for a fixed amplitude $\omega_{0}>0$ and $M>2$ equi-distributed phases $0 \leq \theta_{1}<\ldots<\theta_{M}<2 \pi$.

Introducing the control space $U:=L^{2}\left(0, T ; \mathbb{R}^{2}\right)$, the controlto-state operator

$$
S: U \rightarrow\left(\mathbb{R}^{3}\right)^{J}, \quad u \mapsto\left[\mathbf{M}^{\left(\omega_{1}\right)}(T), \ldots, \mathbf{M}^{\left(\omega_{J}\right)}(T)\right],
$$

* CC is supported by the German Science Fund (DFG) under grant CL 487/1-1. CT gratefully acknowledges support by the DFG Research Training Group 2088 Project A1. BW's research was supported by the Alfried Krupp Prize for Young University Teachers awarded by the Alfried Krupp von Bohlen und Halbach-Stiftung. The work was also supported by the Deutsche Forschungsgemeinschaft (DFG), Cells-in-Motion Cluster of Excellence (EXC1003 - CiM), University of Münster, Germany. and the target vector $z:=\left[\mathbf{M}_{d}^{\left(\omega_{1}\right)}, \ldots, \mathbf{M}_{d}^{\left(\omega_{J}\right)}\right]^{T} \in\left(\mathbb{R}^{3}\right)^{J}$, this problem can be formulated as an optimal control problem

$$
\min _{u \in U} \frac{1}{2}\|S(u)-z\|_{2}^{2}+\int_{0}^{T} g(u(t)) \mathrm{d} t .
$$

Here, $g: \mathbb{R}^{2} \rightarrow[0, \infty]$ is a vector-valued version of the multi-bang penalty from Clason and Kunisch (2014, 2016) that can be motivated as the convex hull of the non-convex penalty $\frac{\alpha}{2}|\cdot|_{2}^{2}+\delta_{\mathcal{M}}$, where $\delta_{\mathcal{M}}$ denotes the indicator function in the sense of convex analysis, and can thus be expected to promote controls with values only in $\mathcal{M}$. The main advantage of this formulation is that it leads to a convex optimization problem that can be efficiently solved using a semismooth Newton method (Clason et al., 2016).

\section{CONVEX ANALYSIS FRAMEWORK}

Standard arguments from convex analysis and on the continuity and differentiability of $S$ yield the following results.

Theorem 2.1. There exists a solution $\bar{u} \in U$ to (1)

Theorem 2.2. Let $\bar{u} \in U$ be a local minimizer of (1). Then there exists a $\bar{p} \in U$ satisfying

$$
\left\{\begin{array}{c}
-\bar{p}=S^{\prime}(\bar{u})^{*}(S(\bar{u})-z)=: \mathcal{F}^{\prime}(\bar{u}), \\
\bar{u}(t) \in \partial g^{*}(\bar{p}(t)) \quad \text { a.e. } t \in[0, T] .
\end{array}\right.
$$

Here, $S^{\prime}(u)^{*}$ denotes the adjoint of the Fréchet derivative of $S$, which can be computed as the solution of the adjoint Bloch equation, and $\partial g^{*}$ denotes the convex subdifferential of the Fenchel conjugate of $g$, which will be specified below.

For the numerical solution, we also require the MoreauYosida regularization

$$
\left\{\begin{aligned}
-p_{\gamma} & =S^{\prime}\left(u_{\gamma}\right)^{*}\left(S\left(u_{\gamma}\right)-z\right)=\mathcal{F}^{\prime}\left(u_{\gamma}\right) \\
u_{\gamma}(t) & =h_{\gamma}\left(p_{\gamma}(t)\right) \quad \text { a.e. } t \in[0,1]
\end{aligned}\right.
$$

where

$$
h_{\gamma}:=\left(\partial g^{*}\right)_{\gamma}=\frac{1}{\gamma}\left(\mathrm{Id}-\operatorname{prox}_{\gamma g^{*}}\right)
$$

and $\operatorname{prox}_{\gamma g^{*}}=\left(\operatorname{Id}-\gamma \partial g^{*}\right)^{-1}$ denotes the (single-valued and Lipschitz-continuous) proximal mapping of $g^{*}$. 


\section{MULTIBANG PENALTY}

Letting $\bar{u}_{i}, i=0, \ldots, M$, denote the admissible control values in $\mathcal{M}$ (with $\bar{u}_{0}=0$ ), we obtain from the definition of the Fenchel conjugate and the maximum rule for the subdifferential by straightforward if tedious computation the following explicit characterization for $q:=p(t)$ :

$$
\partial g^{*}(q)= \begin{cases}\left\{\bar{u}_{i}\right\} & q \in Q_{i} \\ \operatorname{co}\left\{\bar{u}_{i_{1}}, \ldots, \bar{u}_{i_{k}}\right\} & q \in Q_{i_{1} \ldots i_{k}}\end{cases}
$$

Together with (2) this yields that apart from singular cases corresponding to the second line, the optimal control $\bar{u}$ will indeed take on values from the admissible set. The sets $Q_{i}$ can be characterized using polar coordinates; here we only illustrate their distribution in Fig. 1a.

Similarly, we obtain for the Moreau-Yosida regularization

$$
h_{\gamma}(q)= \begin{cases}0 & q \in Q_{0}^{\gamma}, \\ \bar{u}_{i} & q \in Q_{i}^{\gamma}, \\ \left(\frac{\left\langle q, \bar{u}_{i}\right\rangle}{\gamma \omega_{0}^{2}}-\frac{\alpha}{2 \gamma}\right) \bar{u}_{i} & q \in Q_{0, i}^{\gamma}, \\ \frac{\bar{u}_{i}+\bar{u}_{i+1}}{2}+\frac{\left\langle q, \bar{u}_{i}-\bar{u}_{i+1}\right\rangle\left(\bar{u}_{i}-\bar{u}_{i+1}\right)}{\gamma\left|\bar{u}_{i}-\bar{u}_{i+1}\right|_{2}^{2}} & q \in Q_{i, i+1}^{\gamma}, \\ \frac{q}{\gamma}-\frac{\alpha}{\gamma}\left(\frac{\omega_{0}}{\left|\bar{u}_{i}+\bar{u}_{i+1}\right|_{2}}\right)^{2}\left(\bar{u}_{i}+\bar{u}_{i+1}\right) & q \in Q_{0, i, i+1}^{\gamma},\end{cases}
$$

with the subdomains $Q_{i}^{\gamma}$ illustrated in Fig. $1 \mathrm{~b}$.

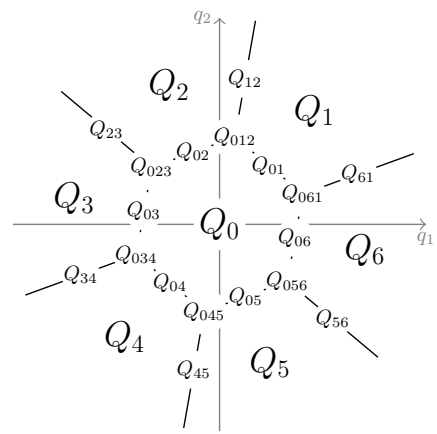

(a) $\partial g^{*}$

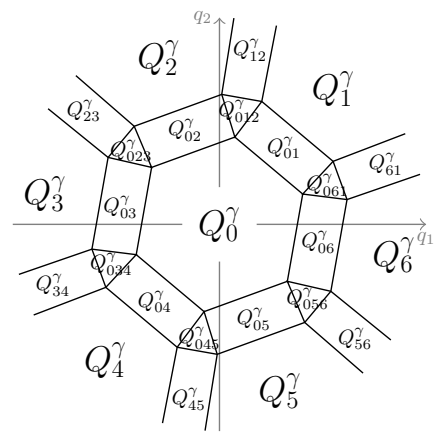

(b) $h_{\gamma}$
Fig. 1. Subdomains for radially distributed $\mathcal{M}, M=6$

\section{NUMERICAL SOLUTION}

Introducing the superposition operator $H_{\gamma}: U \rightarrow U$ via $\left[H_{\gamma}(p)\right](t)=h_{\gamma}(p(t))$, we can write $(3)$ in reduced form as

$$
u_{\gamma}-H_{\gamma}\left(\mathcal{F}^{\prime}\left(u_{\gamma}\right)\right)=0 \text {. }
$$

Since $h_{\gamma}$ is Lipschitz continuous and piecewise differentiable and the range of $S^{\prime}(u)^{*} \subset L^{\infty}\left(0, T ; \mathbb{R}^{2}\right)$, this is a Newtondifferentiable equation. Taking

$$
D_{N} h_{\gamma}(q)= \begin{cases}0 & q \in Q_{i}^{\gamma}, \\ \frac{\bar{u}_{i} \bar{u}_{i}^{T} 1}{\gamma \omega_{0}^{2}} & q \in Q_{0, i}^{\gamma}, \\ \frac{\left(\bar{u}_{i}-\bar{u}_{i+1}\right)\left(\bar{u}_{i}-\bar{u}_{i+1}\right)^{T}}{\gamma\left|\bar{u}_{i}-\bar{u}_{i+1}\right|_{2}^{2}} & q \in Q_{i, i+1}^{\gamma}, \\ \frac{1}{\gamma} \mathrm{Id} & q \in Q_{0, i, i+1}^{\gamma},\end{cases}
$$

the corresponding superposition operator $D_{N} H_{\gamma}(p)$ leads to a superlinearly convergent semismooth Newton method

$$
\left(\operatorname{Id}-D_{N} H_{\gamma}\left(\mathcal{F}^{\prime}\left(u^{k}\right)\right) \mathcal{F}^{\prime \prime}\left(u^{k}\right)\right) \delta u=-u^{k}+H_{\gamma}\left(\mathcal{F}^{\prime}\left(u^{k}\right)\right),
$$

which can be realized using a matrix-free Krylov method such as GMRES (where $\mathcal{F}^{\prime \prime}(u)$ can be computed using the solution of two linearized (adjoint) Bloch equations).

\section{NUMERICAL EXAMPLES}

Figure 2 illustrates the above approach for the simultaneous control of $J=4$ isochromats with from $\mathbf{M}_{0}=(0,0,1)^{T}$ to $\mathbf{M}_{d}^{\left(\omega_{j}\right)}=(0,0,1)$ for $j=3, \mathbf{M}_{0}$ else, and $M=6$ admissible values with equidistant phases (marked with dashed lines in Fig. 2a), where the implementation of the discrete (linearized) Bloch and adjoint equations is taken from Aigner et al. (2015). For more details and examples, see Clason et al. (2016).

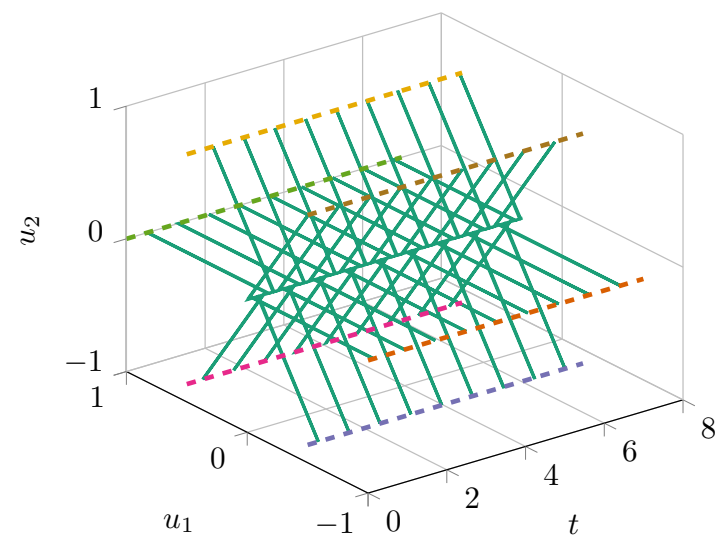

(a) control $\tilde{u}(t)$

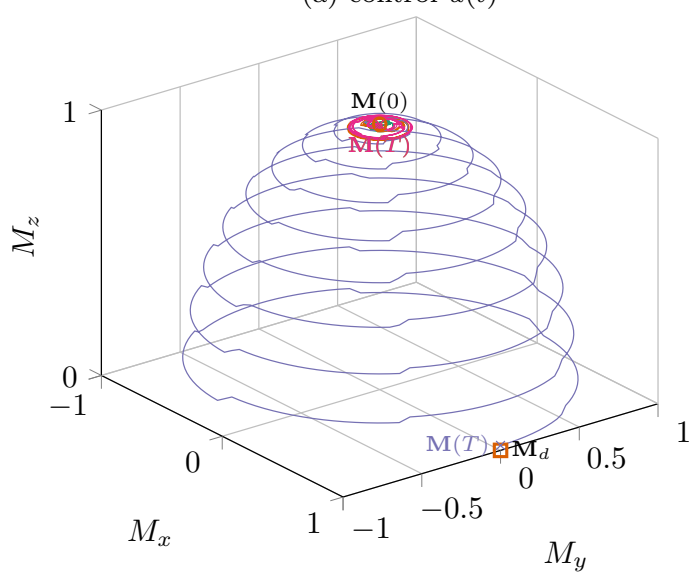

(b) state $\mathbf{M}_{u}^{\left(\omega_{j}\right)}(t)$

Fig. 2. Optimal control and state for $M=6, J=4$

\section{REFERENCES}

Aigner, C.S., Clason, C., and Rund, A. (2015). rfcontrol. doi:10.5281/zenodo.31187.

Clason, C. and Kunisch, K. (2014). Multi-bang control of elliptic systems. Annales de l'Institut Henri Poincaré (C) Analyse Non Linéaire, 31(6), 1109-1130. doi:10.1016/j.anihpc.2013.08.005.

Clason, C. and Kunisch, K. (2016). A convex analysis approach to multi-material topology optimization. ESAIM: Mathematical Modelling and Numerical Analysis, 50(6), 1917-1936. doi:10.1051/m2an/2016012.

Clason, C., Tameling, C., and Wirth, B. (2016). Vectorvalued multibang control of differential equations. arXiv. URL https: //arxiv .org/abs/1611.07853.

Dridi, G., Lapert, M., Salomon, J., Glaser, S.J., and Sugny, D. (2015). Discrete-valued-pulse optimal control algorithms: Application to spin systems. Physical Review A, 92(4), 043417. doi:10.1103/PhysRevA.92.043417. 